\title{
Les momies du Muséum national d'Histoire naturelle : du cabinet anthropologique au musée de l'Homme
}

The National Natural History Museum's Mummies: from the anthropological cabinet to the Museum of Mankind

\section{Pauline Carminati}

\section{(2) OpenEdition}

\section{Journals}

Édition électronique

URL : http://journals.openedition.org/ocim/966

DOI : $10.4000 /$ ocim. 966

ISSN : 2108-646X

Éditeur

OCIM

Édition imprimée

Date de publication : 1 septembre 2011

Pagination : 26-34

ISSN : 0994-1908

\section{Référence électronique}

Pauline Carminati, « Les momies du Muséum national d'Histoire naturelle : du cabinet anthropologique au musée de l'Homme », La Lettre de l'OCIM [En ligne], 137 | 2011, mis en ligne le 01 septembre 2013, consulté le 19 avril 2019. URL : http://journals.openedition.org/ocim/966 ; DOI 10.4000/ocim.966 


\section{Les momies du Muséum national d'Histoire naturelle : \\ du cabinet anthropologique au musée de l'Homme}

\section{Pauline Carminati *}

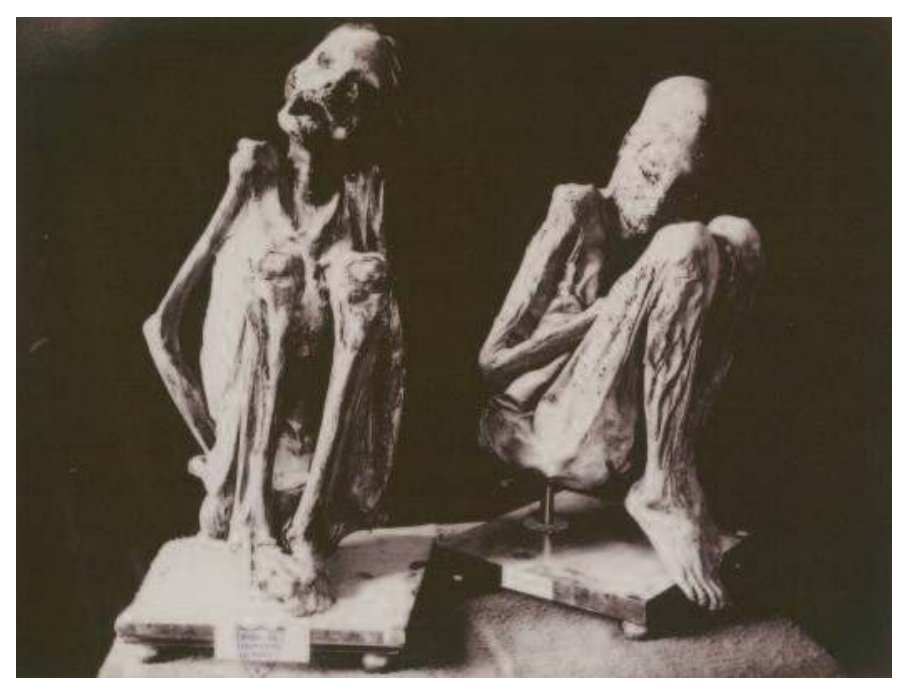

Deux momies andines de la collection anthropologique du Muséum dans les années 1890.

À gauche, la momie qui a fait l'objet de notre mémoire. () Bibliothèque centrale du MNHN/Pierre Petit

* Pauline Carminati est restauratrice du patrimoine paulinecarminati@yahoo.fr
En s'appuyant sur l'histoire générale de I'Anthropologie, l'auteur retrace le parcours de la collection de momies du Muséum national $d^{\prime}$ Histoire naturelle, revenant sur son étude, les conditions de sa conservation, les choix effectués pour sa présentation et montrant que ce type de collection historique conserve encore aujourd'hui un intérêt scientifique.

Le musée de l'Homme conserve un important ensemble de corps humains momifiés : soixante-cinq spécimens complets et de nombreux fragments provenant d'Égypte, d'Amérique du Nord, d’Amérique du Sud, de France et des Canaries. Cet ensemble fait partie des collections d'Anthropologie physique attachées au laboratoire d’Anthropologie du Muséum national d'Histoire naturelle. Il est conservé depuis 2002 au sein d'une plateforme dirigée par Anne Raggi, restauratrice du patrimoine. Remisées dans les réserves, ces momies n'ont fait l'objet que de très rares études et leur histoire dans les collections est encore peu documentée. À l'occasion d'un mémoire de recherche en conservation-restauration, différents aspects de leur patrimonialisation ont pu être mis en lumière ${ }^{(1)}$.

L'actuelle collection du laboratoire d'Anthropologie est issue de celle qui a été fondée en 1839 au Muséum pour former le «cabinet anthropologique » de la nouvelle chaire d'Anatomie et d'Histoire naturelle de l'Homme (future chaire d'Anthropologie). Après la création du musée de l'Homme en 1937, sont venus s'ajouter à ce noyau des dons, échanges et acquisitions, ainsi que le dépôt de la collection du musée Broca (musée de la Société d'Anthropologie de Paris) en $1950^{(2)}$. 
L'histoire de l'Anthropologie en France a déjà fait l'objet de nombreuses études et le contexte institutionnel et scientifique de la création de la chaire d'Anthropologie au Muséum est maintenant bien connu (3). L'histoire de la collection afférente à cette chaire a également été étudiée à plusieurs reprises. Dès le XIXe siècle, les professeurs et leurs aides-naturalistes en ont décrit la formation, l'accroissement et la présentation au public (4). Plus récemment, Claude Blanckaert et Nélia Dias notamment se sont penchés sur le contexte de sa création et sur les liens qui l'unissent aux théories anthropologiques (5). Cependant, le rôle des momies dans la collection n'a jamais fait l'objet d'une étude spécifique. Dans quelle mesure participaient-elles au développement de l'Anthropologie, motivant leur entrée dans la collection anthropologique du Muséum au XIXe siècle ? Quelles ont été les modalités particulières de leur étude, de leur présentation et de leur acquisition? Comment a évolué leur rapport avec le reste de la collection?

Au regard des registres d'inventaires, les momies ne représentent qu'une infime partie de la collection anthropologique telle qu'elle s'est constituée au cours du XIXe siècle au Muséum. En nombre beaucoup plus restreint que les squelettes, moulages ou photographies, et rarement incluses dans les mentions du contenu de la collection, elles semblent n'avoir présenté qu'un intérêt moindre pour les anthropologues de l'époque. Le dépouillement des périodiques du Muséum parus au XIXe siècle révèle qu'aucune étude ne leur a alors été consacrée. Au cours de la même période, les publications personnelles des professeurs d'Anthropologie ne s'attardent pas davantage sur ce sujet. Il apparaît donc que la place des momies au sein de la collection anthropologique était relativement marginale. Les mentions de ces spécimens dans les sources bibliographiques étant limitées à quelques rares évocations insérées dans des études plus générales, les corps eux-mêmes et les traces qu'ils portent des pratiques anthropologiques passées ont constitué des sources d'information de premier ordre.

\section{Éfude et conservation}

En 1852, Étienne Serres, titulaire de la chaire d'Anatomie et d'histoire naturelle de l'Homme, encourageait « l'exploration des sépultures anciennes et modernes, afin de rassembler les restes des peuples qui ont existé ». Son successeur, Armand de Quatrefages, exprimait le même intérêt en 1860 : «Dans les contrées où se sont succédées des populations diverses, on recherchera avec soin les momies, squelettes et crânes des populations disparues ou dispersées ». Il engageait donc également à fouiller «toutes les anciennes sépultures dans l'ancien aussi bien que dans le nouveau continent».

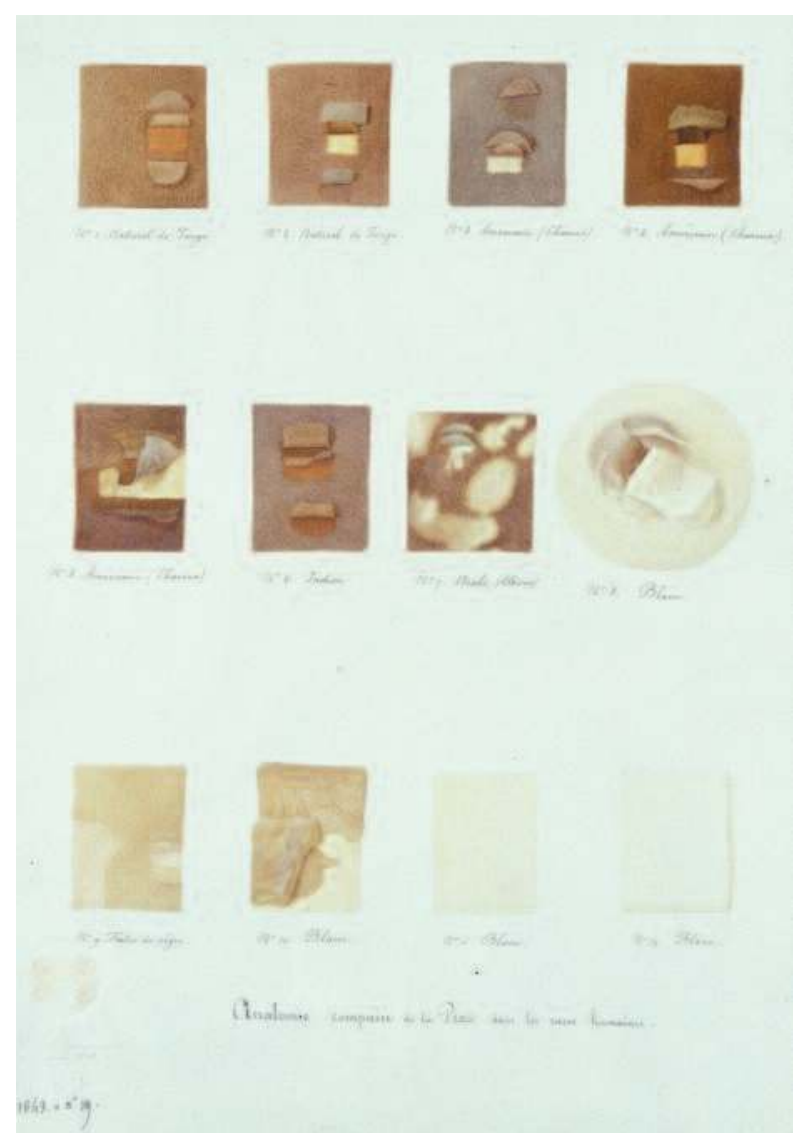

Anatomie comparée de la peau dans les races humaines, vélin de Jean-Charles Werner, 1843. La couleur de la peau était au milieu du XIXe siècle un des critères de comparaison et de classification des races humaines sur lesquels s'appuyaient les anthropologues, en premier lieu Étienne Serres dont JeanCharles Werner était l'un des précieux collaborateurs. (c) Bibliothèque centrale du MNHN

Les momies, au même titre que les ossements, représentaient les peuples anciens dans l'étude générale des « races humaines » telle qu'elle définissait alors l'Anthropologie, et étaient utilisées pour de nombreuses comparaisons. La forme et les dimensions des os, en particulier celles du crâne et du bassin, la couleur de la peau et de l'iris, la couleur et la texture des cheveux étaient les critères principaux sur lesquels les anthropologues du Muséum se sont appuyés pour caractériser et distinguer différentes races, classer les grandes familles du genre humain et éclaircir la question des migrations antiques et $\mathrm{du}$ " mélange des races ».

Dans la perspective de l'évolution d'un type primitif en plusieurs variétés et de leurs mélanges, les caractères physiques étaient également représentatifs de l'histoire des races. Selon Serres, l'«étude comparée des nuances de la peau » apporte « des données probables sur l'origine des populations » en déterminant «à quelle variété de races ou de mélanges de races se rapportent les colorations observées ». Cet intérêt porté à l'origine des peuples, en particulier l'origine des populations d'Amérique demeurée longtemps 
mystérieuse a contribué à la multiplication et à la systématisation des fouilles, liant étroitement archéologie et anthropologie.

Afin de faciliter les comparaisons, les nuances des cheveux, de la peau et de l'iris étaient rassemblées dans des tableaux où elles sont reproduites à la peinture d'après modèle ou représentées directement par des échantillons. Quatrefages indiquait à ce sujet que «les chevelures fournissant à l'ethnologie quelques-unes de ses données les plus précises, on devra chercher à les recueillir. Les cheveux coupés avec soin sur des individus vivants seront mis en boîte en évitant de les presser ». Pour représenter les peuples anciens, des échantillons étaient prélevés sur les momies, comme en témoignent les registres d'inventaire et les corps eux-mêmes qui portent les traces de ces prélèvements de peau et de cheveux. Ces échantillons étaient présentés avec un grand nombre d'autres échantillons de races différentes et formaient la « collection spéciale », utilisée pour «compléter les résultats obtenus à l'aide du daguerréotype et du moulage».

Outre les prélèvements, les méthodes d'étude comprenaient souvent le retrait de la peau des momies dans le but d'effectuer des mesures plus précises du squelette. Nombre de squelettes de momies servaient ainsi de pendants aux squelettes modernes. La tête était également parfois séparée du reste du corps pour les études craniologiques. Ces pratiques traduisent parfaitement la place de premier plan qu'occupaient le crâne et le squelette, jusqu'au début du XXe siècle. D’une manière générale, elles révèlent que les momies, comme tous les spécimens réunis par et pour les anthropologues du Muséum, étaient avant tout des objets d'étude : toute intervention était justifiée du point de vue de l'enseignement et de la recherche "dans l'intérêt du progrès scientifique ». Les professeurs étaient unanimes : "Reconnaissant en principe que l'institution du Muséum est scientifique avant tout, nous nous élèverons toujours contre l'opinion de ceux qui, ne voyant absolument que la conservation, prétendraient que les objets renfermés dans les galeries doivent y être immuables [...]. Les collections du Muséum, créées pour la science, doivent servir autant que possible à l'étudiant et au savant».

Mais la conservation dans le temps n'était pour autant pas négligée par les professeurs qui considéraient la collection comme une réserve d’informations, formée en prévision de futures recherches. Ainsi, dans la mesure où les objets étaient fréquemment sortis de leur vitrine, emportés dans les laboratoires pour être étudiés et remis en place, les socles et les potences servaient de supports et facilitaient la manipulation et le déplacement d'objets fragiles tout en les protégeant de contacts répétés. Les problèmes posés par la conservation d'une collection en majorité composée de matières organiques, en particulier face à la menace des insectes kératophages, n'étaient pas ignorés. De nombreuses recettes visant à détruire ces insectes ou à prévenir leur attaque sont rapportées dans les manuels de préparation anatomique et dans des ouvrages spécialisés. Ces publications témoignent de la préoccupation constante que représentait déjà la conservation de ce type de collection. Au Muséum, un vernis épais à base de résine mastic ou dammar, dilué dans la térébenthine, a été appliqué sur plusieurs momies pour stopper une attaque d'insectes ou, dans certains cas, pour la prévenir.

La collection anthropologique apparaît donc comme un matériel d'étude réuni pour répondre aux besoins de l'étudiant et du savant. La nécessité de former une telle collection a été revendiquée dès 1838 par Flourens. Un an plus tard, à sa nomination, Serres demandait une galerie spéciale pour permettre " le rassemblement des types des peuples et des races, dans un même lieu et sur un même point». Ainsi " au lieu d'aller à la recherche des peuples, ce qui est impossible à un seul homme, ce seront les peuples qui, à certains égards, iront à la rencontre de l'observateur ». Les collections réunies étaient toutes destinées à être exposées dans ce que Serres nommait au départ le « cabinet anthropologique ». Prêtes à la fin de l'année 1839, les salles mises à sa disposition ne seront ouvertes au public qu'en 1855. Aménagées dans un ancien logement de fonctionnaire au premier étage de la galerie d'Anatomie comparée de Cuvier, elles se révélèrent rapidement insuffisantes et sept autres pièces furent ajoutées en 1855, puis deux autres avant 1875. C'est cette «enfilade de petites pièces carrelées en rouge » qui forma la galerie anthropologique jusqu'en 1898, date à laquelle les collections furent transférées dans un nouveau bâtiment (celui des actuelles galeries d'Anatomie comparée et de Paléontologie).

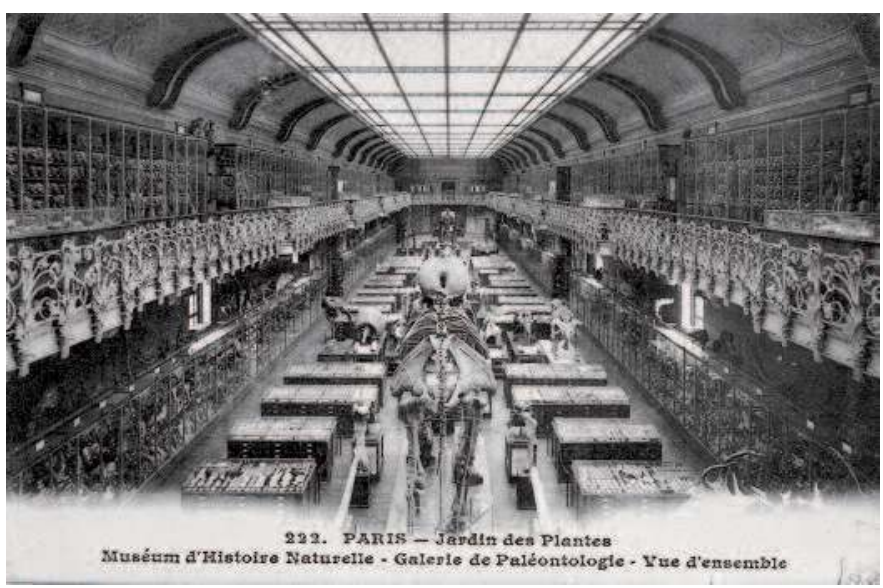

Galerie de Paléontologie et d'Anthropologie du Muséum national d'Histoire naturelle en 1910. Les momies étaient regroupées dans quelques-unes des vitrines basses logées dans les avancées du balcon, et placées aux côtés d'objets ethnographiques. () Bibliothèque centrale du MNHN 


\section{Choix de présentation}

La constitution de la collection fut définie dès le départ sur le modèle des sciences naturelles : Serres entendait créer "un musée des races humaines et de leurs variétés, formé d'après les bases qui ont présidé aux collections $d u$ Muséum d'Histoire naturelle ». Son projet muséologique s'affirmait en continuité avec l'approche méthodologique de la zoologie et de la botanique, comparatiste et classificatrice. Par conséquent, l'idée de série a guidé la formation de la collection, qui tendait toujours plus vers l'accumulation. L'« encombrement » qui en résulta était d'ailleurs un problème constant pour les administrateurs : les galeries étaient combles et une partie de la collection devait être conservée en réserve, dans les greniers.

Les objets accumulés pour la recherche et l'enseignement ont été généralement présentés de manière à favoriser la compréhension des approches successives de l'Anthropologie. Bien que la collection soit principalement destinée au savant et à l'étudiant, le public devait aussi pouvoir saisir un discours à travers la muséographie.

L'organisation choisie par Serres illustrait les théories développées dans ses cours par la comparaison des quatre grandes divisions de l'espèce humaine : les races caucasique, mongole, américaine et éthiopique. Déramond, alors aide-naturaliste de la chaire, rapporte en effet que, dès 1855, « ces quatre races sont représentées dans les galeries par des crânes, des squelettes, des photographies, des tableaux et des bustes moulés sur nature et coloriés. [...] Il faut ajouter des momies égyptiennes, des momies indiennes, des momies pérwviennes, des momies américaines, des momies guanches, des momies gauloises ».

Contrairement à Serres, Quatrefages semble assez indifférent à l'ordonnance des collections dont il a eu la charge. Aucun témoignage n'indique qu'il a modifié l'organisation des salles de son prédécesseur et, dans les salles supplémentaires acquises en 1855, il aurait fait disposer les collections «à peu près au hasard». Mais en 1873, à son arrivée comme aide-naturaliste, Ernest-Théodore Hamy les réorganisa en suivant un classement géographique.

En 1878, Paul Topinard décrivit la collection anthropologique telle qu’elle était alors présentée au public (6). Il indique par exemple que «la troisième salle est entièrement consacrée à la magnifique collection de crânes égyptiens recueillis par MM. Mariette et Prisse » et que "quatre momies $d u$ moyen et du nouvel empire les accompagnent ». Il poursuit : « La quatrième salle est encore consacrée à l'Égypte. Elle renferme 19 momies $[\ldots] ; 1$ momie d'Axoumite dans son coffre funéraire; 1 momie d'enfant; 6 squelettes montés; de nombreux portraits à l'huile [...]; 6 bustes d'Abyssins moulés sur le vivant, etc. ». Il rapporte ensuite qu'au centre de la sixième salle, dédiée à l'Amérique du Sud, « on

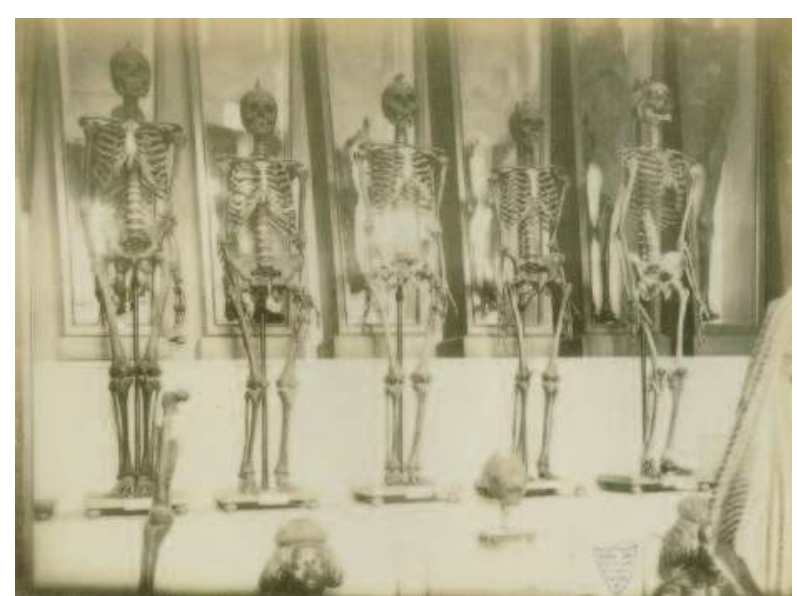

Squelettes de momies égyptiennes dans la quatrième salle de la galerie d'Anthropologie du Muséum vers 1880. En arrière plan, les momies débandelettées dans des vitrines en forme de cercueil. Momies, squelettes de momies et squelettes modernes, moulages et photographies étaient placés en regard dans les salles de la galerie d'Anthropologie. (c) Bibliothèque centrale du MNHN/Pierre Petit

voit 6 momies de Quichua et d'Aymara ». À leurs côtés sont exposés de nombreux crânes et squelettes, " 6 têtes préparées par les Mundurucus; enfin une momie péruvienne de Chancaï dont toute la sépulture a été reconstituée par M. Hamy ». Cette description correspond à l'état dont témoignent les photographies retrouvées dans les archives du Muséum.

En 1898, dans la nouvelle galerie, la distribution des spécimens dut s'adapter à l'espace exigu affecté à l'Anthropologie. En effet, les collections représentant les « races actuelles » prenaient place sur un balcon qui surplombait la galerie de Paléontologie. La majorité des vitrines, alignées contre le mur, étaient donc hautes et étroites. Y étaient exposées les séries de crânes et de squelettes, classées par race, des plus «inférieures » aux «supérieures »: en entrant, le visiteur commençait par les « races nègres », puis les « races jaunes», les « races américaines » et à la fin du parcours, les «races blanches». En revanche, les momies des divers pays (Égypte, Éthiopie, Bolivie, Pérou, Canaries) étaient quant à elles présentées dans des vitrines basses indépendantes, en association avec des objets ethnographiques. Les descriptions de cette galerie mettaient en avant le manque de place : Verneau, alors aidenaturaliste de la chaire, rapporte dès 1898 que «la place attribuée à l'anthropologie est si insuffisante qu'une partie des collections logées dans le vieux bâtiment de la rue Cuvier n'ont pu tenir dans le nouveau musée. Toutes les séries importantes ne sont représentées que par un nombre relativement restreint de pièces dans la galerie nouvelle. Le reste ira grossir le stock déjà considérable d'objets conservés dans les magasins, qui seront sans doute trop petits dès aujourd'hui. » 
Les quelques mentions et photographies conservées et présentées ici traduisent l'évolution générale du regard porté sur les momies au Muséum. Les deux premières descriptions (1855 et 1878) indiquent qu'elles étaient alors intégrées au classement général où elles étaient directement mises en parallèle avec les crânes, squelettes et moulages. Les momies andines étaient présentées sur un socle avec une étiquette et dépourvues de tout vêtement. De la même façon, les momies égyptiennes étaient présentées débandelettées, dans des vitrines individuelles en forme de cercueil. À la différence de ce mode de présentation, l'une des momies de la salle consacrée à l'Amérique du Sud était accompagnée de textiles et d'offrandes pour représenter les conditions de découverte dans le tombeau. Cette reconstitution, qui date de 1874, témoigne de l'apparition au Muséum d'une approche plus ethnographique, valorisant les productions matérielles et soucieuse de présenter des éléments de contexte aux côtés des pièces d'étude exclusivement anatomique. Topinard précise qu'Ernest-Théodore Hamy, alors aide-naturaliste, en est à l'origine : son rôle dans l'essor et l'institutionnalisation de l'Ethnographie est en effet très important. Le musée d'Ethnographie du Trocadéro sera créé à son initiative en 1878 et restera sous sa direction jusqu'à sa mort en 1908.

Cette attention nouvelle évolue en une séparation complète avec les séries ethniques dans la nouvelle galerie de 1898. Désormais, les momies ne participent plus aux comparaisons raciales au même titre que les squelettes, elles sont qualifiées de "séries à part » et présentées indépendamment. Dans la salle principale, alors que les squelettes sont alignés en une longue et unique file faisant le tour d'un balcon, respectant le classement géographique antérieur, les momies sont quant à elles regroupées dans de petites vitrines logées dans les avancées du balcon et mises en rapport avec des objets ethnographiques. Si le manque de place et la disposition des vitrines ne sont sans doute pas étrangers à cette rupture muséographique, on peut également y voir la conséquence plus profonde d'une évolution de l'Anthropologie.

Quelques hypothèses peuvent être émises pour éclairer ce changement, amorcé dès l'arrivée d'Hamy en 1873. À partir de cette date et jusqu'en 1882, Quatrefages et Hamy entamèrent une série de travaux craniologiques sur les races humaines dont l'ouvrage Crania Ethnica fut l'aboutissement. L'Anthropologie a alors été marquée par une attention particulière accordée au crâne sec, au détriment d'autres caractères (peau, cheveux...) représentés notamment par les momies. Il semble en outre que les restes momifiés n'étaient pas adaptés aux méthodes d'étude anthropologique : la craniologie impliquait des observations et des mesures très précises, qui ne pouvaient pas s'effectuer avec les chairs desséchées, et le développement des séries,

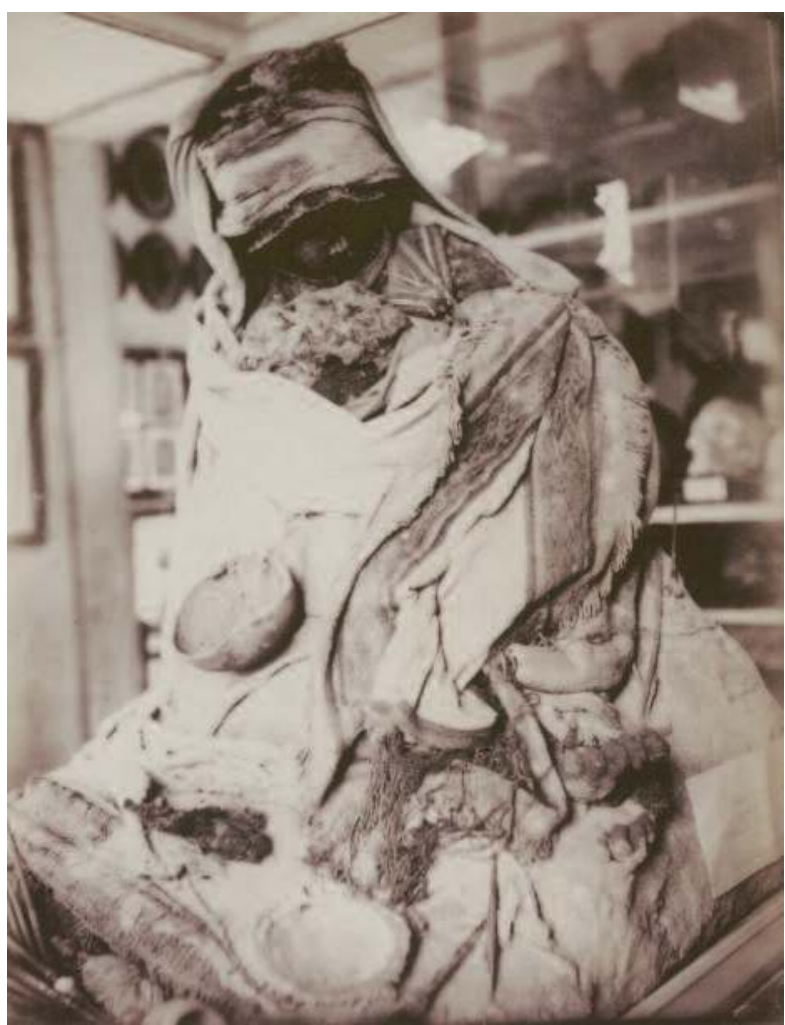

Reconstitution de la sépulture d'une momie andine dans une vitrine de la sixième salle de la galerie d'Anthropologie du Muséum vers 1880. Avec l'arrivée d'Ernest-Théodore Hamy au Muséum, les années 1870 voient émerger une approche ethnographique des momies. (๑) Bibliothèque centrale du MNHN/Pierre Petit

" seul moyen de parvenir à des conclusions d'une valeur générale », s'appliquait uniquement aux crânes et aux squelettes. L'intérêt anatomique des momies semble donc avoir diminué au profit d'un intérêt ethnographique, au moment où l'Ethnographie commençait à se distinguer de l'Anthropologie et à se constituer comme discipline autonome ${ }^{(7)}$.

L'évolution des acquisitions rend compte d'un tel changement d'approche : dès 1878, les momies nouvellement découvertes, contrairement aux crânes et aux squelettes, ne sont plus pour la plupart envoyées au Muséum mais au musée d'Ethnographie du Trocadéro, où elles sont présentées systématiquement avec les objets et les vêtements qui ont été trouvés et rapportés avec elles. Il est particulièrement significatif qu'Hamy, quand il était directeur de la chaire d'Anthropologie et du musée d'Ethnographie, ne traitât pas de la même manière les momies selon leur destination : lorsque les lots du musée d'Ethnographie contenaient plusieurs momies, il en préparait une en squelette pour enrichir la collection du Muséum et seules les mieux conservées étaient présentées dans les galeries du musée d'Ethnographie. Celles-ci faisaient alors l'objet d'études spécifiques ${ }^{(8)}$. 
Plus tard, au musée de l'Homme, la plus grande partie des salles d'exposition a été consacrée aux collections du musée d'Ethnographie du Trocadéro. Henri Vallois précise que «malgré l'importance des documents d'anthropologie physique, deux seules salles dans le musée proprement dit leur sont consacrées. Il n'y avait, en effet, aucun intérêt à montrer au public, comme cela se fait dans trop de musées, des séries de crânes et de squelettes alignés à perte de vue dans une suite de vitrines » (9). D'après les photographies conservées au musée du quai Branly, seules deux momies d'enfants et une momie de femme adulte étaient exposées dans ces deux salles consacrées à l'Anthropologie. Les autres momies du Muséum ont été directement mises en réserve à leur arrivée.

Depuis la création de la collection anthropologique jusqu'au milieu de la seconde moitié du XIXe siècle, les momies ont fait partie des spécimens dignes d'intérêt qu'il fallait se procurer et, malgré un net ralentissement à partir des années 1880, elles sont entrées dans la collection tout au long du siècle. En réalité, il semblerait que les professeurs étaient, dans une large mesure, tributaires de voyageurs non spécialistes et ce mode d'acquisition laissait peu de place à un choix raisonné. La collection s'est donc constituée au gré des circonstances, dans un but d'accroissement privilégiant, selon certains, la quantité à la qualité. L'acquisition des momies apparaît ainsi sans grande cohérence. L'étude des conditions dans lesquelles ces acquisitions ont été réalisées apporte certains éclaircissements sur les méthodes de collecte et les difficultés rencontrées, qui permettent de mieux comprendre la place des momies dans la collection.

\section{Acquisition}

En parcourant les registres d'inventaire, on constate que la grande majorité des momies du Muséum, tout comme les squelettes, ont fait l'objet de dons. En effet, le budget extrêmement réduit de la chaire d'Anthropologie ne pouvant couvrir que les frais de fonctionnement et d'entretien, "il ne reste que bien peu de chose pour les achats proprement dits ». Les professeurs ne voyageant pas pour se procurer directement les spécimens dont ils avaient besoin, l'accroissement de la collection anthropologique reposait en grande partie sur les dons, faits à titre personnel ou à l'occasion d'une expédition officielle financée par le gouvernement.

Les dons étaient donc encouragés activement : les nombreuses instructions aux voyageurs publiées tout au long du XIXe siècle par le Muséum, par les sociétés savantes ou par le gouvernement, mais également les revues de vulgarisation scientifique, "entretinrent et diffusèrent l'idée selon laquelle il incombait à chacun de participer à un effort scientifique collectif, pour contribuer au progrès de l'humanité » (10). Le patriotisme était également un argument largement employé dans ces publications, ainsi dans les instructions du Muséum en 1860 : «ce ne sont pas de simples renseignements que nous donnons ici, c'est un appel que nous faisons à tous ceux qui s'intéressent à la science et à la grandeur de leur pays ». En contrepartie, les noms des donateurs étaient "soigneusement recueillis dans [les] archives et indiqués à la reconnaissance publique sur les étiquettes mêmes annexées aux objets apportés par eux ».

L'absence de professionnels sur le terrain et le manque de préparation des voyageurs aboutissant à de fréquentes déceptions quant à l'intérêt et/ou l'état de conservation des objets rapportés, les différents professeurs de la chaire d'Anthropologie ont été amenés à formuler précisément la nature de leurs besoins. Lorsqu'une expédition se préparait, Serres adressait des recommandations précises sur la nature des observations à effectuer sur place et sur les pièces qu'il convenait de rapporter. Quatrefages publia également des instructions dans un ouvrage édité par le Muséum en 1860 et sollicitait directement les diplomates séjournant à l'étranger afin qu'ils se procurent des pièces intéressantes et les envoient au Muséum. Les institutions devenant de plus en plus exigeantes dans les observations demandées et dans la manière de recueillir objets et informations, des conférences pour les voyageurs ont été proposées à partir de 1876. Hamy précise que "chacun des partants était exactement renseigné sur la bibliographie anthropologique des pays qu'il allait visiter, l'état des collections spéciales qui y avaient été formées, les recherches particulières qu'il y avait lieu d'y poursuivre ».

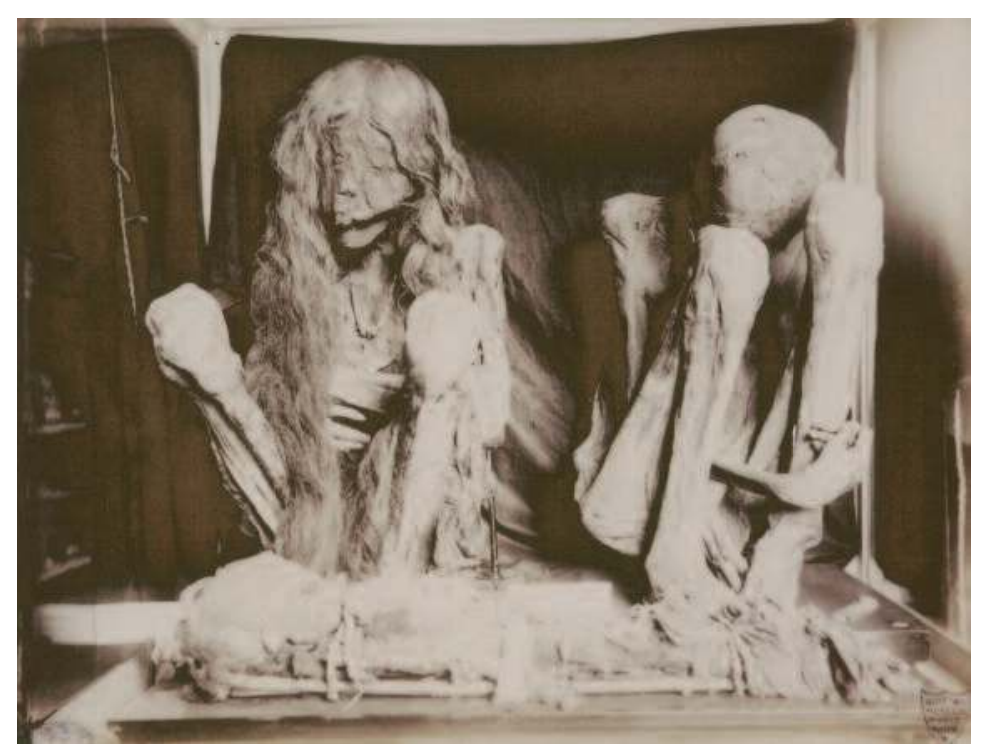

Trois momies andines dans une vitrine de la sixième salle de la galerie d'Anthropologie du Muséum vers 1880 . Les momies étaient présentées sur des socles. Les étiquettes rappelaient le nom du donateur, le numéro d'inventaire et la provenance du spécimen. (c) Bibliothèque centrale du MNHN/Pierre Petit 
Le socle a été, au XIX ${ }^{\mathrm{e}}$ siècle et pendant une grande partie du XXe siècle, une constante dans la muséographie des momies. Cette pratique unifiait une collection composée d'objets de diverses natures et constituait une véritable signature. Ainsi, chaque collection présentait des socles distinctifs, qui lorsqu'ils sont encore conservés permettent d'identifier l'origine des objets.

Au musée de l'Homme, les socles des pièces exposées ont été remplacés par de nouveaux pour s'accorder à la nouvelle muséographie et rompre avec un style qui rappelait sans doute trop les cabinets de curiosités. Toutefois, les momies qui n’ont pas été destinées à l'exposition dans les galeries ont conservé leur socle d'origine et constituent des témoins très intéressants de ces pratiques passées.
Aujourd'hui les anciens soclages sont souvent retirés. D'une manière générale, de nouveaux critères régissent la présentation des momies dans les musées en accord avec le Code de Déontologie de l'ICOM et les articles concernant les restes humains. Une reconstitution de la tombe est parfois proposée, comme cela a été fait en 1985 au British Museum pour la momie égyptienne surnommée « Ginger ». Quelques musées choisissent de mettre en avant l'intérêt historique des présentations anciennes qui témoignent à la fois de la formation d'une collection et d'un état particulier de l'histoire muséographique. C'est le cas par exemple du musée du Cinquantenaire à Bruxelles.
Il apparaît au travers des témoignages laissés par les donateurs, lettres adressées au Muséum ou récits de voyage, que les momies offertes au cours du XIXe siècle sont souvent des découvertes fortuites, intervenues lors de fouilles ayant d'autres buts (par exemple la Paléontologie), ou lors de travaux (aménagement ou exploitation du sol). Généralement, les donateurs n'effectuaient pas eux-mêmes les fouilles mais achetaient les pièces qui les intéressaient, conscients de l'intérêt qu'elles pouvaient représenter et désireux, suivant Pascal Riviale, "de signaler leur "zèle" face à une mission de portée nationale ».

Parfois cependant, certains voyageurs passionnés, ou des chargés de missions officielles, entreprenaient des fouilles, mais celles-ci étaient "menées sans méthode, à seule fin de recueillir des objets entiers »; rares en effet furent les relevés effectués sur le terrain, et les descriptions accompagnant parfois les objets manquent toujours de précision. Aussi, dans la seconde moitié du XIXe siècle, des critiques ont-elles été formulées en faveur d'une archéologie plus scientifique.

Néanmoins, le don de momies était un événement assez rare. Les difficultés étaient multiples, qu'elles soient d'ordre politique, cultuel ou matériel. Les fouilles étaient difficiles à réaliser en Amérique du Sud par exemple, car les Indiens s'y opposaient fréquemment, luttant en particulier contre le pillage des tombes de leurs ancêtres et le prélèvement de leurs restes, squelette ou momie. Le transport des momies jusqu'en France semble également avoir représenté une difficulté importante, particulièrement les traversées en mer. En 1843, le médecin de marine Liautaud rapportait : "Plusieurs de mes collègues ont tenté à diverses reprises de transporter en France quelques-unes de ces momies péruviennes. Mais malgré les précautions les plus minutieuses ils n'ont jamais pu les soustraire à l'humidité de l'atmosphère intérieure du navire qui pénètre ces tissus spongieux au bout de peu de temps et les transforme en une espèce de boue informe et déliquescente. Les précautions hygiéniques que nécessitait la santé de l'équipage confié à mes soins, m'ont forcé à me défaire de mes pièces anatomiques au bout d'un temps fort court et de ne conserver que les crânes, après les avoir fait exactement nettoyer ».

La prédominance des crânes et des squelettes pour l'Anthropologie de la seconde moitié du XIXe siècle, la séparation progressive de l'Anthropologie et de l'Ethnographie, les difficultés à se procurer des spécimens bien conservés, tous ces éléments contribuent à expliquer que seul un petit nombre de momies soit entrée dans les collections du Muséum et que peu d'études leur aient alors été consacrées. Le musée de l'Homme a néanmoins hérité d'un nombre important de corps momifiés. Avec le développement des fouilles archéologiques, de nouvelles acquisitions sont venues enrichir ce fonds ancien dans la seconde moitié du $\mathrm{XX}$ e siècle. Cet ensemble, pour lequel a été créé un service spécifique en 2002, est en cours d'étude et de valorisation.

\section{Mise en valeur}

L'étude des momies issues d'anciennes collections se heurte aujourd'hui à un double problème. D'une part, une longue conservation dans les musées, dans des conditions peu ou pas contrôlées, a souvent entraîné une perte de données, tant sur le plan biologique (les contaminations, les démembrements, la putréfaction...) que sur le plan historique (les données attachées aux numéros d’inventaire, la documentation...), les informations collectées au XIXe siècle étant par ailleurs extrêmement pauvres, peu précises et soumises à caution. D'autre part, l'utilisation scientifique 
actuelle de ce type de collection est limitée par la perte du contexte archéologique, l'exploitation des résultats dépendant de la possibilité de rattacher le spécimen à une culture donnée.

Néanmoins, plusieurs auteurs ont montré qu'une « recontextualisation » est possible et par conséquent que ces collections historiques peuvent toujours présenter un intérêt scientifique (11). Ainsi, l'étude présentée dans cet article s'est intégrée dans un processus plus global de recontextualisation, appliqué à l'une des momies andines du musée de l'Homme qui nous a été confiée pour sa restauration et dont le numéro d'inventaire n'était plus connu. Les recherches historiques ont été associées à des observations précises du corps, à des analyses (tomodensitométrie et reconstitutions 3D, datation radiocarbone, identification des dépôts minéraux et organiques présents sur le corps), à une étude bioarchéologique et à des interventions de conservation-restauration, l'ensemble ayant été organisé à partir d'un protocole méthodologique.

Les nombreux résultats recueillis ont démontré la pertinence de cette méthode, fondée sur la complémentarité de différentes disciplines : outre l'historique de cette momie (donateur, conditions d'entrée et parcours dans les collections), la provenance, la datation, l'affiliation culturelle, le traitement funéraire, les transformations taphonomiques, le sexe et l'âge ont pu être identifiés, alors qu'aucune information n'était conservée. Ces données, ainsi que la restauration proprement dite, permettent désormais de revaloriser ce spécimen dans son contexte de conservation à la fois muséal et scientifique.

\section{Conclusion}

La mise en valeur des momies conservées au musée de l'Homme nécessite des recherches historiques approfondies. En l'absence d'une documentation spécialisée, les inventaires, l'observation des spécimens et les analyses scientifiques peuvent apporter de nombreuses informations. Ces données matérielles attendent néanmoins d'être analysées et intégrées aux recherches des historiens de l'Anthropologie afin de mieux comprendre la collection telle qu'elle est aujourd'hui conservée.

L'auteur souhaite remercier Michelle Lenoir, directrice des bibliothèques et de la documentation du MNHN, et Alice Lemaire, responsable du service du patrimoine de la bibliothèque centrale du MNHN, pour leur aimable autorisation de reproduction des photographies issues du fonds iconographique ancien.

\section{Notes}

(1) Carminati, P. Étude et conservation : problématiques de la restauration d'une momie andine des collections du musée de l'Homme, mémoire de diplôme en conservation-restauration, 2010, 307 p. Ce mémoire est consultable à la bibliothèque de l'École supérieure des Beaux-Arts de Tours et à celle de l'INP, département restaurateurs.

(2) Jusqu'en 2001 venaient s'ajouter les collections du musée d'Ethnographie du Trocadéro, attachées au laboratoire d'Ethnologie du musée de l'Homme. Elles sont maintenant conservées au musée du quai Branly, sauf celles d'Europe et d'Afrique du Nord qui seront transférées au musée de l’Europe et de la Méditerranée à Marseille.

(3) Rappelons quelques repères : en 1793, la chaire d'Anatomie et de Chirurgie du Jardin du Roi se divise en deux, la chaire d'Anatomie humaine d'une part et la chaire d'Anatomie comparée d'autre part. Le titulaire de la première fut Antoine Portal de 1793 à 1832. Après que son utilité ait été remise en question, la chaire passe sous la direction de Pierre Flourens jusqu'en 1839. Si elle conserve le même titre, le contenu en est alors complètement modifié. Claude Blanckaert situe avec l'arrivée de Flourens «l'officialisation d'un genre technique nowveau au Muséum, à savoir l'Histoire naturelle de l'homme » et la consécration institutionnelle de l'Anthropologie. Avant de quitter la chaire, Flourens demande la modification officielle du titre et du programme de l'ancien cours d'Anatomie. La chaire prend alors le nom de chaire d'Anatomie et d'Histoire naturelle de l'Homme, d'après les deux composantes fondamentales de l'enseignement de l'Anthropologie. Étienne-Renaud-Augustin Serres est nommé à sa direction en 1839. Il poursuit la réorientation de la chaire et assure "l'autonomie de la science anthropologique ». Il va également constituer une riche collection et obtenir une galerie pour son exposition. Armand de Quatrefages lui succède en 1855. La chaire prend alors le nom de chaire d'Anthropologie. Les professeurs suivants seront Ernest-Théodore Hamy, de 1892 à 1908, René Verneau, de 1908 à 1928, et Paul Rivet qui organisa la création du musée de l'Homme.

(4) Serres, E.-R.-A. Cabinet anthropologique du Muséum d'Histoire naturelle, Nouvelles annales des voyages et des sciences géographiques, t. 6, n4, 1853, pp. 358-360 ; Déramond, É. Leçon d’Anthropologie sur les caractères généraux distinctifs de l'espèce humaine ; par M. Serres. Nouvelles galeries au Muséum d'Histoire naturelle, Gazette Médicale de Paris, t. 10, n³0, 1855, pp. 463-465 ; Verneau, R. Les nouvelles galeries du Muséum. La galerie d'anthropologie, L'Anthropologie, t. 9, 1898, pp. 327-336 ; Hamy, E.-T. La collection anthropologique du Muséum national d'Histoire naturelle, L'Anthropologie, t. 18, 1907, pp. 257-276.

(5) Blanckaert, C. La création de la chaire d’Anthropologie du Muséum dans son contexte institutionnel et intellectuel (1832-1855), in Blanckaert, C. Le Muséum au premier siècle de son histoire. Paris : Muséum national d'Histoire naturelle, 1997, pp. 85-125 ; Dias, N. Séries de crânes et armées de squelettes : les collections anthropologiques en France dans la seconde moitié du XIXe siècle, Bulletins et mémoires de la Société d'Anthropologie de Paris, vol. 1, n³-4, 1989, pp. 203-230 ; Dias, N. Le musée d'Ethnographie du Trocadéro, anthropologie et muséologie en France. Paris : CNRS, 1991, 310 p. 
(6) Topinard, P. Visite à la collection anthropologique du Muséum d'Histoire naturelle. Le dimanche 18 août 1878, in Congrès international des sciences anthropologiques tenu à Paris du 16 an 21 août 1878, Exposition universelle, Paris 1878. Paris : Imprimerie nationale, 1880, pp. 293-298.

(7) Voir Hamy, E.-T. Les origines du musée d'Ethnographie. Histoire et documents. Paris : Leroux, 1890, 321 p. ; Dias, N. Le musée d'Ethnographie du Trocadéro, anthropologie et muséologie en France. Paris : CNRS, 1991, $310 \mathrm{p}$.

(8) Voir par exemple les études figurant dans Hamy, E.-T. Galerie américaine du musée d'Ethnographie du Trocadéro : choix de pièces archéologiques et ethnographiques décrites et publiées par le Dr E.-T. Hamy. Paris : Leroux, 1897, 118 p.

(9) Vallois, H.-V. Le musée de l'Homme, Médecine de France, n71, 1956, pp. 17-32.
(10) Riviale, P. Un siècle d'archéologie française au Pérou (1821-1914). Paris/Montréal : L’Harmattan, 1996, 425 p.

(11) Par exemple : Arriaza, B.-T., Cartmel, L.-L., Moragas, C. et al. The bioarchaeological value of human mummies without provenience, Chungará, vol. 40, $\mathrm{n}^{\circ} 1,2008$, pp. 55-65 ; Madden, G.-D. Recontextualization and affiliation assessment of un-provenienced mummified human remains, in Atoche, P., Rodriguez, C. et Ramirez, A. Mummies and Science, World Mummies Research, Proceedings of the VI World Congress on Mummy Studies. Santa Cruz de Tenerife : Beautell, 2008, pp. 89-95.
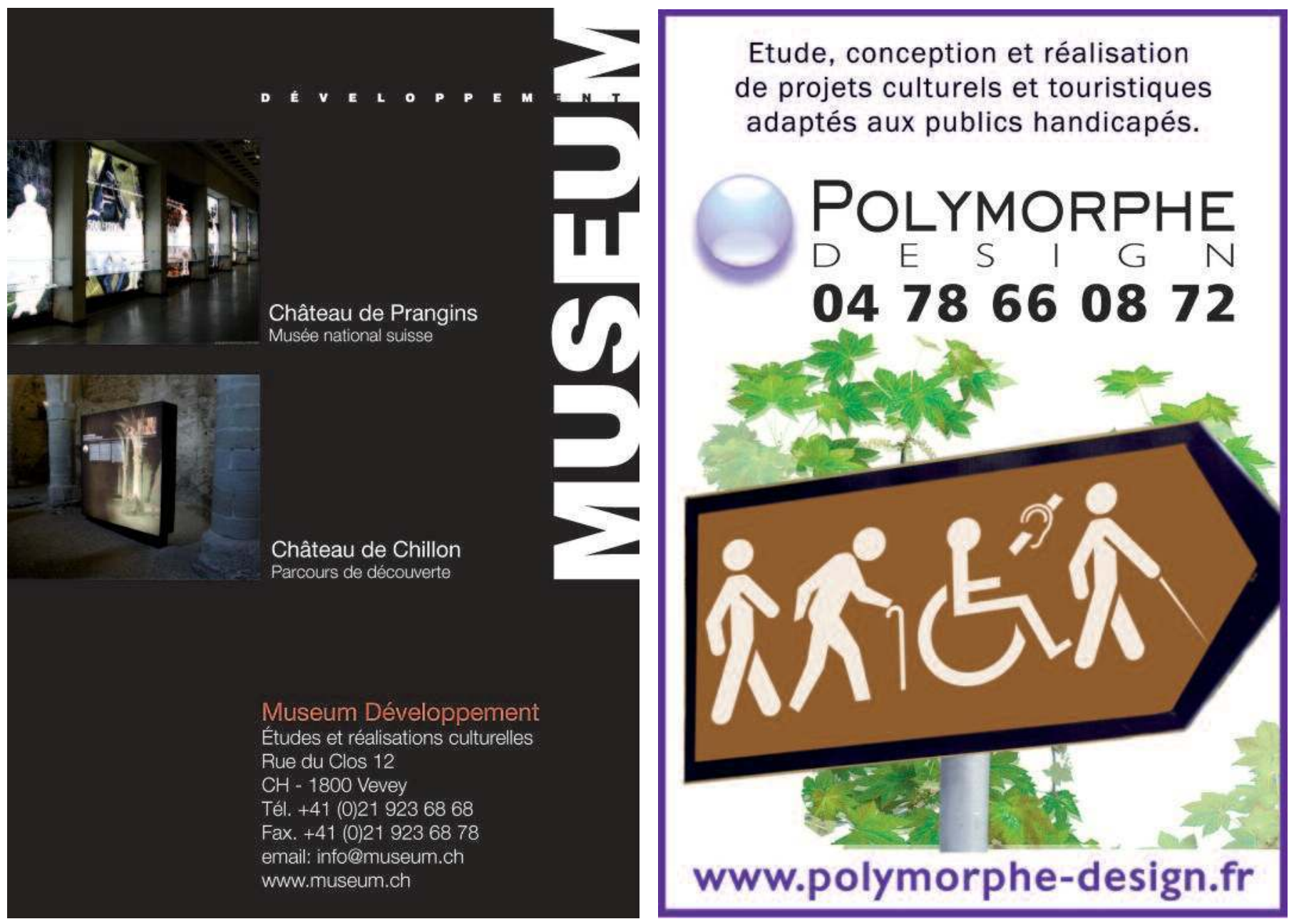